\title{
LA CONTRATACión PROACTIVA DENTRO DE LAS NUEVAS VISIONES DEL CONTRATO*
}

\section{PROACTIVE CONTRACTING WITHIN THE NEW VIEWS OF THE AGREEMENT}

\author{
Mariana Bernal-Fandiño** \\ Fecha de recepción:27 de noviembre de 2014 \\ Fecha de aceptación: 30 de diciembre de 2014 \\ Disponible en línea: 30 de junio de 2015
}

\section{Para citar este artículo/To cite this article}

\author{
Bernal-Fandiño, Mariana, La contratación proactiva dentro de las \\ nuevas visiones del contrato, 130 Vniversitas, 21-52 (2015). http://dx.doi. \\ org/10.11144/Javeriana.vj130.cpdn \\ doi:10.11144/Javeriana.vj130.cpdn
}

\footnotetext{
* Artículo de reflexión

** Abogada, Pontificia Universidad Javeriana. Máster en derecho comercial y máster en derecho internacional privado, Universidad de París II. Doctora en ciencias jurídicas, Universidad Javeriana. Profesora investigadora, Universidad Sergio Arboleda.

Contacto: Mariana.bernal@usa.edu.co
} 


\section{RESUMEN}

Este artículo es producto del proyecto de investigación Debates contemporáneos alrededor del negocio jurídico. Con este texto, se pretende revisar algunas de las discusiones más relevantes acerca del contrato y su funcionalidad en la sociedad actual a partir de visiones diferentes, como el análisis económico del derecho, el solidarismo, los contratos relacionales y la contratación sostenible, entre otras. Se analiza el movimiento de la contratación proactiva, en general y en particular, se estudia un caso de la regulación de comunicaciones en Colombia como manifestación de esta propuesta. Se propone que la aplicación de la contratación proactiva cambia el paradigma de la contratación tradicional para dar respuesta a las inquietudes planteadas.

Palabras clave: contratación proactiva; visualización y simplificación de los contratos; dinamismo contractual; función social del contrato; contratación sostenible 


\section{ABSTRACT}

This article is based on the research project Contemporary Debates on the Legal Business. The purpose of this text is to review some of the most relevant discussions on the contract and its functionality in our current society from different points of view such as the economic analysis of law, solidarism, relational contracts, and sustainable contracting, among others. We analyze the movement of proactive contracting in general terms and also in a particular case, as we study the communications regulation in Colombia as an expression of this proposal. We suggest that the application of proactive contracting changes the paradigm of traditional contracting as an answer to the proposed questions.

Keywords: proactive contracting; visualization and simplification of contracts; contractual dynamism; social function of contracts; sustainable contracting

\section{SUMARIO}

Introducción.- I. Funciones de los contratos.- $A$. Función de los contratos desde su noción jurídica.- B. Función del contrato desde una perspectiva jurídico-económica.- C. Función social de los contratos. D. Contratación sostenible.- E. Función dinámica del contrato.- II. FUNCIÓN PROACTIVA DEL CONTRATO.- A. El derecho preventivo como génesis de la función proactiva del contrato.- B. La visualización de los contratos.- C. Caso simplificación de contratos celulares por parte de la CRC.- ConCLusiones.- Bibliografía. 


\section{INTRODUCCIÓN}

Comparar el derecho normativo con el derecho en acción en materia de contratos lleva a la revisión de la dogmática jurídica y de la forma de hacer los negocios. Los contratos deben ser instrumentos eficientes de intercambio de bienes y servicios y no meras formalidades que, dada su complejidad actual, han sido relegados a un mal necesario al cual acudir si se presenta un conflicto entre los contratantes.

Con su visión dogmática, el derecho contractual tradicional tiende a concentrarse más en la formación del contrato para prever dificultades futuras que en la función que debe cumplir entre los contratantes para facilitar el negocio y permitir que las partes realicen sus intereses y finalidades.

La contratación contemporánea ha sido objeto de diversos cuestionamientos que se reflejan en críticas y visiones diferentes de lo que debería ser el contrato. Estas visiones generalmente se refieren a la función misma del contrato en la sociedad. De esta manera, se puede analizar el contrato desde un punto de vista dogmático, a partir de su definición jurídica o desde una visión jurídico-económica del derecho que tenga en cuenta su función dentro del mercado. También se ha estudiado el contrato desde una visión social que de una forma más amplia se centra en el rol que tiene en la sociedad y las posibilidades de intervención del Estado en los contratos. Con el desarrollo del derecho ambiental, su relación con los contratos cobra cada vez mayor relevancia por medio de la llamada contratación sostenible. La función del contrato como un instrumento de dinamismo en los negocios por oposición a una aproximación tradicionalmente estática de él es otra aproximación interesante que si bien puede tener un ángulo interno puesto que se preocupa por las relaciones de los contratantes en el tiempo, previene a la vez posibles intervenciones del Estado en circunstancias imprevisibles que pongan en riesgo el equilibrio de los negocios.

Con estas diferentes aproximaciones del contrato, en este artículo se pretende analizar en un primer momento la función del contrato en la sociedad desde distintas visiones para identificar los problemas de la contratación contemporánea en cuanto a su funcionalidad (I) 
y presentar la llamada contratación proactiva como una posible solución a los problemas identificados (II).

\section{FUNCIONES DE LOS CONTRATOS}

Tradicionalmente, se ha hablado de la función económico-jurídica del contrato. Sin embargo, en el derecho contemporáneo es necesario tener en cuenta otras visiones del contrato que influyen en su funcionalidad.

\section{A. Función de los contratos desde su noción jurídica}

La definición clásica del contrato establece que se trata de un acuerdo de voluntades para la creación de obligaciones. Mientras la convención sería el género, pues es un acuerdo para la creación, modificación o extinción de relaciones jurídicas, el contrato sería una especie de este género en virtud del cual solamente se generan obligaciones ${ }^{1}$. Desde esta postura, la función del contrato sería meramente la de crear relaciones jurídicas.

El Código Civil colombiano establece en su artículo 1495 que "contrato o convención es un acto por el cual una parte se obliga para con otra a dar, hacer o no hacer alguna cosa"2. Esta definición ha sido muy criticada en la medida en que se acerca más a la definición de obligación que a la de contrato. De la literalidad del artículo podría inicialmente concluirse que la norma equipara las nociones de contrato y convención. Sin embargo, un análisis desde una interpretación más omnicomprensiva del resto de las normas del Código puede conducir a una explicación diferente.

En efecto, del análisis de otras normas, podríamos inducir que en realidad el artículo 1495 está indicando que el contrato, como especie de convención, genera obligaciones de dar, hacer o no hacer. Esto puede explicarse dado que, en otros apartes del Código, en

1 Guillermo Ospina-Fernández, Teoría general del contrato y de los demás actos jurídicos o negocios jurídicos, 43 (4ª ed., Editorial Temis, Bogotá, 1994).

2 Código Civil, Ley 57 de 1887. Disponible en: http://www.secretariasenado.gov.co/senado/ basedoc/codigo_civil.html 
casos de extinción de relaciones jurídicas, las normas se refieren a la convención y no al contrato ${ }^{3}$.

En el Código de Comercio colombiano, el artículo 864 expresa que "el contrato es un acuerdo de dos o más partes para constituir, regular o extinguir entre ellas una relación jurídica patrimonial"4.

De la definición legal que se le ha dado al contrato en la legislación colombiana, podemos concluir que su función será la de generar relaciones jurídicas o, con una visión más amplia, la de generar, modificar o extinguir este tipo de relaciones.

Además de una aproximación puramente dogmática de la noción de contrato, esta ha sido estudiada desde una perspectiva jurídicoeconómica.

\section{B. Función del contrato desde una perspectiva jurídico-económica}

Francesco Messineo señala que el contrato tiene como función la de "ser el centro de la vida de los negocios". El contrato se considera una herramienta de intercambio de bienes y servicios, el medio principal de interacción del mercado por el cual se realizan diversas finalidades de la vida económica. Al hacer previsibles las conductas de los sujetos, el contrato genera confianza en las transacciones y facilita de esta manera la interacción social y económica ${ }^{6}$.

La economía, al ser una ciencia que estudia las decisiones racionales de las personas frente a unos recursos que son escasos y las necesidades que estas quieren satisfacer, ha estudiado el contrato

3 El artículo 1625 del Código Civil, por ejemplo, hace referencia a la convención extintiva exclusivamente, ya no se hace alusión al contrato sino a la convención. "Artículo 1625. Toda obligación puede extinguirse por una convención en que las partes interesadas, siendo capaces de disponer libremente de lo suyo, consientan en darla por nula. Las obligaciones se extinguen además en todo o en parte: 1. Por la solución o pago efectivo. 2. Por la novación. 3. Por la transacción. 4. Por la remisión. 5. Por la compensación. 6. Por la confusión. 7. Por la pérdida de la cosa que se debe. 8. Por la declaración de nulidad o por la rescisión. 9. Por el evento de la condición resolutoria. 10. Por la prescripción”. Código Civil, Ley 57 de 1887. Disponible en: http://www.secretariasenado.gov.co/senado/basedoc/codigo_civil.html

4 Código de Comercio, Decreto 410 de 1971. Disponible en: http://www.alcaldiabogota.gov.co/ sisjur/normas/Norma1.jsp?i=41102

5 Francesco Messineo, Doctrina general del contrato, Tomo I, 34 (Rodolfo Fontanarrosa, Santiago Sentis-Melendo \& M. Volterra, trads., Ediciones Jurídicas Europa-América, Buenos Aires, 1952).

6 Luis Carlos Plata-López, La naturaleza social y económica del contrato, 23 Revista de Derecho, Universidad del Norte, 97-111 (2005). Disponible en: http://www.redalyc.org/articulo. oa?id=85102305 
analizándolo por ejemplo a través de la teoría de los costes de transacción o la teoría de juegos.

El contrato resulta un medio para la reducción de incertidumbres y costos de transacción (la teoría de los costos de transacción se encuentra en la investigación de autores como Ronald H. Coase ${ }^{7}$, George Stigler ${ }^{8}$ y Friedrich August von Hayek ${ }^{9}$ ). Bajo este supuesto, los particulares solo deberían utilizar el contrato si ofrece ventajas competitivas respecto de otros mecanismos que reduzcan los costes. Si los contratos no reducen los costes de transacción sino que se convierten en herramientas pesadas que no facilitan el intercambio, serán sustituidos por otros mecanismos menos eficientes con la consecuente pérdida de bienestar social. Desde este punto de vista, los contratos deben tener como función la solución de problemas de información y la disminución de los costos de transacción ${ }^{10}$.

Acerca de la relación entre la teoría de juegos y el derecho de los contratos, las normas jurídicas en materia contractual se han equiparado a las reglas de un juego productivo pues el contrato supone que mediante la colaboración y las estrategias conjuntas se alcanzan ventajas para todos los participantes. Si las partes cooperan y cumplen de forma efectiva sus acuerdos, se desvirtúa el dilema del prisionero ${ }^{11}$.

Recordemos que en el clásico dilema del prisionero ${ }^{12}$, los prisioneros A y B están separados en diferentes celdas e incomunicados. Las autoridades les ofrecen una salida. Si confiesan e incriminan

7 Ronald H. Coase, The Problem of Social Cost, 3 Journal of Law and Economics, 1-44 (1960). Disponible en: http://www2.econ.iastate.edu/classes/tsc220/hallam/Coase.pdf

8 George Stigler, The Economics of Information, 69 The Journal of Political Economy, 3, 213225 (June, 1969). Disponible en: http://home.uchicago.edu/ vlima/courses/econ200/spring01/ stigler.pdf

9 Friedrich August von Hayex, Economics and Knowledge, 4 Economica, New Series, 13, 33-54 (February, 1937). Disponible en: http://www.econlib.org/library/NPDBooks/Thirlby/bcthLS3. html

10 Hans-Bernd Schäfer \& Claus Ott, Manual de análisis económico del derecho, 322 (Editorial Tecnos, Madrid, 1991). Sobre el análisis del costo de la información, Anthony T. Kronman, Mistake, Disclosure, Information, and the Law of Contracts, 7 Journal of Legal Studies, 1, 1-34 (1978). Disponible en: http://www.rwi.uzh.ch/lehreforschung/alphabetisch/weberr/archiv/ FS08/unterlagen/unterlagenLE/aufsaetze/MistakeDisclosureInformationandtheLawofContracts.pdf. Sobre la relación entre economía y contratos, RoBert CoOTER \& THOMAs Ulen, Law and Economics (Pearson/Addison-Wesley, Boston, 2004).

11 Hans-Bernd Schäfer \& Claus Ott, Manual de análisis económico del derecho, 317 (Editorial Tecnos, Madrid, 1991).

12 Wayne Eastman, How Coasean Bargaining Entails a Prisoners' Dilemma, 72 Notre Dame Law Review, 1, 89-101, 89 (1996). Disponible en: http://scholarship.law.nd.edu/ndlr/vol72/iss1/3/ 
al otro recibirán una disminución en su sentencia. La disminución dependerá de si el otro confiesa o no. Así, si A confiesa y B no lo hace, A recibirá un año de prisión. Si B confiesa también, ambos recibirán 4 años de prisión. Si A no confiesa y B sí lo hace, A recibirá 10 años de prisión. Si ninguno confiesa, cada uno recibirá 2 años.

Tabla 1. El dilema del prisionero

\begin{tabular}{|c|c|c|}
\hline & $\begin{array}{c}\text { Prisionero A } \\
\text { Confiesa }\end{array}$ & $\begin{array}{c}\text { Prisionero A } \\
\text { Silencio }\end{array}$ \\
\hline Prisionero B & A: 4 años & A: 10 años \\
Confiesa & B: 4 años & B: 1 año \\
\hline Prisionero B & A: 1 año & A: 2 años \\
Silencio & B: 10 años & B: 2 años \\
\hline
\end{tabular}

Fuente: elaboración propia

En materia de contratos, a diferencia de lo que sucede con el dilema del prisionero, si ambas partes colaboran para el éxito del negocio, ambas partes realizarán adecuadamente sus intereses.

Esta idea de cooperación, desde otra óptica, ha dado lugar al movimiento solidarista de los contratos que tiene como base estudiarlos desde su función social.

\section{Función social de los contratos}

Hay diversas acepciones de la función social de los contratos. Desde una perspectiva solidarista, se ha considerado que:

(...) la función social del contrato está relacionada con el vínculo de solidaridad, de colaboración que debe existir entre los contratantes. Para el solidarismo, el contrato tiene una función social que debe cumplir en el sentido de que los contratantes deben ejercer la autonomía privada dentro de unos parámetros de equilibrio, cambio justo y armonía. El contrato debe ser un mecanismo de cooperación para lograr así un acuerdo libre, útil y justo ${ }^{13}$.

13 Mariana Bernal-Fandiño, El deber de coherencia en el derecho colombiano de los contratos, 56, 57 (Editorial Pontificia Universidad Javeriana, Bogotá, 2013). Disponible en: www.atlantiswordprocessor.com/en/forum/download.php?id=313 
La idea de colaboración entre los contratantes aparece en los desarrollos del principio general de la buena fe. En virtud de la función integradora de este principio, se entienden comprendidos dentro del contenido del contrato ciertos deberes aun sin haber sido expresamente pactados por las partes ${ }^{14}$. Entre estos deberes, llamados colaterales o secundarios de conducta, se encuentran los deberes de información, lealtad, confidencialidad, consejo, protección y coherencia, entre otros.

Autores italianos como Emilio Betti o Massimo Bianca evidencian la relación entre solidaridad y buena fe precisamente por las exigencias que limitan el actuar de los contratantes e imponen la observancia de conductas encauzadas al recto obrar contractual ${ }^{15}$. De forma similar, autores del common law insisten en la importancia de la colaboración entre las partes de los contratos y la observancia de la buena $\mathrm{fe}^{16}$.

Otra forma de entender la función social de los contratos va más allá de la colaboración que se deben los contratantes pues se refiere a la posibilidad del Estado de intervenir en los negocios de los particulares. Algunos consideran que la visión solidarista de los contratos está basada en una concepción sociológica colectivista e identifican la función social del contrato con "el propósito de equilibrar los poderes económicos y fácticos de las partes, a fin de atender a la finalidad de justicia distributiva propia del Estado Social"17.

El nuevo Código Civil brasileño indica en su artículo 421 que "la libertad de contratar será ejercida en razón y dentro de los límites

14 Sobre los deberes colaterales de conducta, Arturo SolARTE-Rodríguez, La buena fe contractual y los deberes secundarios de conducta, 108 Vniversitas, 281-315 (diciembre de 2004). Disponible en: http://www.javeriana.edu.co/revistas/Facultad/juridicas/universitas/UserFiles/Descargas/ ediciones/108/7_Solarte.pdf

15 Massimo Bianca, Derecho civil, el contrato, T. 3, 444 (Fernando Hinestrosa \& Édgar Cortés, trads., Editorial Universidad Externado de Colombia, Bogotá, 2007). Emilio Betti, Teoría general de las obligaciones, T. 1, 71 (José LuIs De Los Mozos, trad., Editorial Revista de Derecho Privado, Madrid, 1969).

16 Daniel Markovits, Contract and Collaboration, 113 Yale Law Journal, 1417-1518 (2004). Disponible en: http://digitalcommons.law.yale.edu/fss_papers/414/, http://digitalcommons. law.yale.edu/cgi/viewcontent.cgi?article $=1413 \&$ context $=$ fss_papers. THOMAs D. BARTON, Improving Contracts through Expanding Perspectives of Understanding, en Proceedings of the 2013 Academic Forum on Integrating Law and Contract Management: Proactive, Preventive and Strategic Approaches (2013).

17 Luciano Benetti Timm, La función social del derecho contractual en el Código Civil brasileño: justicia distributiva vs. eficiencia económica, 52 Revista de Instituciones, Ideas y Mercados, 5-51, 6(2010). Disponible en: http://www.eseade.edu.ar/files/riim/RIIM_52/52_1_benetti_timm.pdf 
de la función social del contrato"18. Esta disposición ha sido muy discutida pues los alcances de la función social del contrato no son claros, en particular cuando, como se ha comentado, hay diferentes acepciones de este concepto.

Se ha considerado que hay un "cuasiconsenso" en la doctrina brasileña acerca del fundamento que tiene la función social del contrato en la justicia distributiva ${ }^{19}$. El Estado social implica, desde esta perspectiva, la posibilidad de que el Estado intervenga en la autonomía privada para promover el desarrollo económico y social. La voluntad de los individuos no puede abstraerse del entorno social por lo que en los contratos además de elementos consensuales se entienden comprendidos elementos no consensuales ${ }^{20}$. El modelo de contrato que adopta la legislación brasileña, para algunos, es una "cláusula general" que exige que el contrato esté al servicio de la realización del "principio de la socialidad" 21 , lo que implica una manifestación del paradigma sociológico-solidarista, dada su función dentro de la cohesión social.

Al llevar este paradigma a otros ámbitos, se ha encontrado una estrecha relación entre el desarrollo sostenible y la función social de los contratos ${ }^{22}$, lo que resulta interesante particularmente en este texto puesto que la llamada "contratación sostenible" se ha presentado como otra de las visiones del derecho contemporáneo de los contratos.

18 Brasil, Código Civil, Ley 10406 del 10 de enero de 2002. "Art. 421. A liberdade de contratar será exercida em razão e nos limites da função social do contrato". Disponible en: http://www. wipo.int/wipolex/es/details.jsp?id=9615

19 Luciano Benetti Timm, La función social del derecho contractual en el Código Civil brasileño: justicia distributiva vs. eficiencia económica, 52 Revista de Instituciones, Ideas y Mercados, 5-51, 7 (2010). Disponible en: http://www.eseade.edu.ar/files/riim/RIIM_52/52_1_benetti_timm.pdf

20 Resulta clara la relación entre el principio de la buena fe y la función integradora del contenido contractual independientemente de lo acordado expresamente por las partes.

21 Gerson Luiz Carlos Branco, Libertad contractual y su funcionalización: orientación metodológica y lenguaje utilizados por la comisión elaboradora del Código Civil brasileño, 123 Vniversitas, 347-372 (julio-diciembre de 2011). Disponible en: http://www.scielo.org.co/pdf/ vniv/n123/n123a12.pdf

22 Luciana Costa Poli \& Bruno Ferraz Hazan, A função social dos contratos como instrumento para a sustentabilidade, 15 Revista Direito e Liberdade, RDL, ESMARN, 1, 171-193 (2013). Disponible en: http://www.esmarn.tjrn.jus.br/revistas/index.php/revista_direito_e_liberdade/ article/viewFile/550/512 


\section{Contratación sostenible}

La relación entre el derecho de contratos y el derecho ambiental suscita cada vez un mayor interés internacional y nacional. Algunos se han referido incluso a la "función ambiental" de los contratos ${ }^{23}$.

Celebrar contratos teniendo en cuenta criterios ambientales se conoce como contratación sostenible y se ha definido como:

(...) aquel sistema donde se indica a los actores relacionados con las etapas de la contratación, sea esta de carácter público o privado, la incorporación de criterios ambientales en estos, por medio de elementos jurídicamente permitidos y ambientalmente acordes, dentro de los diferentes tipos de contratos establecidos en la legislación, sin contrariar los derechos y responsabilidades de estos y sus entes relacionados en el marco de la gestión integral ${ }^{24}$.

En el ámbito internacional, se deben destacar los esfuerzos de la Organización de Naciones Unidas, ONU, al desarrollar los principios fundamentales y el programa de acción para lograr el desarrollo sostenible que surgieron de la Conferencia de las Naciones Unidas sobre el Medio Ambiente y el Desarrollo, con la Declaración de Río de Janeiro en 1992 ${ }^{25}$. Posteriormente, el Plan de Aplicación de las Decisiones de la Cumbre Mundial sobre el Desarrollo Sostenible, que se realizó en Johannesburgo en 2002, recomendó la adopción de medidas encaminadas entre otras cosas a:

Reunir y difundir información sobre ejemplos de eficacia en función de los costos en producción menos contaminante, eficiencia ecológica y ordenación ambiental, y promover el intercambio de prácticas óptimas y conocimientos

23 Lucas Abreu Barroso, Función ambiental de los contratos agrarios, 1 Rivista di Diritto Agrario, Milano, 190-202 (2005). Disponible en: http://www.abda.com.br/palestrabarroso.htm, http:// www.academia.edu/5427909/Funci\%C3\%B3n_ambiental_de_los_contratos_agrarios

24 Héctor Andrés Moreno-Vásquez, Contratación sostenible, en Perspectivas del derecho ambiental en Colombia, 390-408 (Beatriz Londoño-Toro, Gloria Amparo Rodríguez, Giovanni J. Herrera-Carrascal, eds., Editorial Universidad del Rosario, Bogotá, 2006).

25 Conferencia de las Naciones Unidas sobre el Medio Ambiente y el Desarrollo, Declaración de Río de Janeiro en 1992 sobre el Medio Ambiente y el Desarrollo, 3 al 14 de junio de 1992. Disponible en: http://www.un.org/spanish/esa/sustdev/agenda21/riodeclaration.htm 
especializados en tecnologías racionales desde el punto de vista ambiental entre las instituciones públicas y las privadas ${ }^{26}$.

En el derecho comparado, específicamente en la doctrina brasileña, se ha explicado la función social del contrato como un instrumento que al reflejar ideas solidaristas debe colaborar con el desarrollo sostenible pues no puede verse exclusivamente como un proceso económico de intercambio de bienes y servicios sino que debe además propender por el mejoramiento de la sociedad ${ }^{27}$.

En Colombia, la contratación sostenible es un tema que también está creciendo, principalmente, en el sector público. Sin embargo, compartimos la opinión de quienes consideran que la protección al medio ambiente es una responsabilidad que atañe igualmente al sector privado, pues la propia Constitución Política le ha otorgado a la propiedad una función ecológica y ha establecido el derecho al ambiente $\operatorname{sano}^{28}$.

El Ministerio de Ambiente y Desarrollo Sostenible viene liderando el Plan de Contratación Pública Sostenible en el marco de la Política Nacional de Producción y Consumo Sostenible ${ }^{29}$. Con esta estrategia se busca definir lineamientos que orienten a las entidades públicas para que sus contrataciones beneficien a la institución, la sociedad y la economía al mismo tiempo que se minimice el impacto sobre el medio ambiente.

En Bogotá, por ejemplo, la Secretaría Distrital de Ambiente ha adelantado el Programa de Gestión Ambiental Empresarial ${ }^{30}$ que implementa un sistema de Compras Verdes para ser promovido por

26 Plan de Aplicación de las Decisiones de la Cumbre Mundial sobre el Desarrollo Sostenible, Johannesburgo (Sudáfrica), 2 al 4 de septiembre de 2002. Disponible en: http://www.revistafuturos.info/documentos/johanesburgo/a_conf199_L1.doc

27 Luciana Costa Poli \& Bruno Ferraz Hazan, a função social dos contratos como instrumento para a sustentabilidade, 15 Revista Direito e Liberdade, RDL, ESMARN, 1, 171-193 (2013). Disponible en: http://www.esmarn.tjrn.jus.br/revistas/index.php/revista_direito_e_liberdade/ article/viewFile/550/512

28 Rafael E. Wilches-Durán, Principio ambiental de precaución y contratación mercantil en derecho colombiano. Planteamiento del problema, 123 Vniversitas, 283-313, 290 (2011). Disponible en: http://www.redalyc.org/articulo.oa?id=82522608010, http://vniversitasjuridica.javeriana. edu.co/descargas.php?archivo=Universitas-123\%20-\%209.pdf\&idArt=313\&edicion=123

29 Colombia, Ministerio de Ambiente, Vivienda y Desarrollo Territorial, Política Nacional de Producción y Consumo Sostenible, Ministerio de Ambiente, Vivienda y Desarrollo Territorial, Bogotá (2010). Disponible en: http://www.soyecolombiano.com/site/Portals/0/documents/ PoliticaNacionaldeProduccionyConsumoSostenible.pdf

30 Secretaría Distrital de Ambiente, Programa de Gestión Ambiental Empresarial. Disponible en: http://ambientebogota.gov.co/es/web/gae/inicio 
las entidades del Distrito y los particulares que prestan servicios públicos. Estas compras verdes se han definido como "el proceso mediante el cual las autoridades públicas tratan de adquirir bienes, servicios y obras con un impacto ambiental reducido durante todo su ciclo de vida en comparación con los bienes, servicios y obras con la misma función principal que normalmente se hubiera adquirido" ${ }^{\prime 31}$. De esta manera, la Administración Distrital se compromete a realizar actividades y procesos institucionales con miras a minimizar el impacto ambiental.

Vemos que el contrato, como lo ha afirmado la doctrina, se convierte, mediante este tipo de políticas, en un instrumento para la eficacia de la protección del medio ambiente ${ }^{32}$. En efecto, a pesar de que el contrato es considerado como un instrumento liberal destinado al intercambio de bienes y servicios y por ello alejado, en principio, de la protección de intereses difusos ${ }^{33}$, una mirada distinta desde una finalidad ecológica permite abrirle este camino al derecho de los contratos. Esta perspectiva del contrato sigue la evolución que ha tenido el derecho de propiedad en el derecho colombiano, que desde la Constitución reconoce su función social y ecológica.

\section{E. Función dinámica del contrato}

Otra de las visiones actuales del derecho de los contratos promueve una función más dinámica de los negocios por oposición a una función estática de ellos. El derecho contractual clásico es estático pues se concentra primordialmente en el momento de formación del negocio jurídico ${ }^{34}$.

31 Concejo de Bogotá, Acuerdo 540 de 2013, por medio del cual se establecen los lineamientos del programa distrital de compras verdes y se dictan otras disposiciones, 26 de diciembre de 2013. Disponible en: http://www.alcaldiabogota.gov.co/sisjur/normas/Norma1.jsp?i=56074

32 Mathilde Boutonnet, L'efficacité environnementale du contrat, en L'efficacité du droit de l'environnement - Mise en æuvre et sanctions, 21-46, 34 (Olivera BošKović, dir., Éditions Dalloz, Paris, 2010).

33 Mathilde Boutonnet, L'efficacité environnementale du contrat, en L'efficacité du droit de l'environnement - Mise en æuvre et sanctions, 21-46, 34 (OLIVERA BošKović, dir., Éditions Dalloz, Paris, 2010).

34 Melvin Aron Eisenberg lo llama el modelo estático de derecho contractual por oposición a un modelo más dinámico y flexible. Melvin Aron Eisenberg, The Emergence of Dynamic Contract Law, 88 California Law Review, 6, 1743-1814 (December, 2000). Disponible en: http:// scholarship.law.berkeley.edu/cgi/viewcontent.cgi?article=1470\&context=californialawreview 
El clausulado de un contrato desde una visión clásica generalmente no responde a las reales necesidades de las partes, en particular, si se trata de un contrato de larga duración en el que no se tienen en cuenta los posibles cambios durante su ejecución pues solamente se tienen en cuenta las circunstancias de un momento en el tiempo: el perfeccionamiento del acuerdo.

Hay propuestas doctrinales que buscan dotar de mayor flexibilidad a los contratos de manera que se puedan adaptar a los cambios que se presenten en su vida haciendo énfasis en la planeación contractual ${ }^{35}$. Se ha considerado que las empresas pueden construir alianzas estratégicas empezando por contratos pequeños seguidos por un acuerdo de colaboración continua de forma que los contratantes puedan comprobar sus capacidades y entendimiento antes de entrar en una relación de larga duración ${ }^{36}$.

Entre estas propuestas, queremos resaltar la teoría de los contratos relacionales que surge en el derecho anglosajón para flexibilizar los contratos que se desarrollan durante largos períodos, de modo que puedan evolucionar de acuerdo con las nuevas necesidades que surjan y que no se previeron o no podían preverse en la formación del negocio ${ }^{37}$.

Esta visión contractual se refiere a los vínculos de larga duración, por una parte y por otra, a las redes contractuales ${ }^{38}$. Se busca con esta teoría modificar el régimen contractual que se caracteriza por ser aislado, discontinuo y creado conforme a las necesidades presentes y no futuras para adaptarlo a un proceso continuo y dinámico ${ }^{39}$.

35 Como afirma Pablo Salvador-Coderch: "La renegociación ahorra hoy los costes de prever lo que puede ocurrir mañana o dentro de diez años y permite, en particular, reajustar ex post el contrato a nuevas dificultades surgidas después de su celebración”. PABLo SALVADOR-CODERCH, Alteración de circunstancias en el art. 1213 de la Propuesta de Modernización del Código Civil en materia de Obligaciones y Contratos, 4 InDret, Revista para el Análisis del Derecho, 1-60 (octubre de 2009). Disponible en: http://www.indret.com/pdf/687_es.pdf

36 Larry A. DiMatteo, Strategic Contracting: Contract Law as a Source of Competitive Advantage, 47 American Business Law Journal, 4, 727-794, 736 (2010). Disponible en: http://works. bepress.com/larry_dimatteo/3/

37 Principalmente desarrollada por Ian Roderick MacNeil, The New Social Contract. An Inquiry into Modern Contractual Relations (Yale University Press, New Haven, Connecticut, 1980). Ian Roderick MacNeil, The Many Futures of Contracts, 47 Southern California Law Review, 691-816 (1974).

38 Ricardo Luis Lorenzetti, Problemas actuales de la teoría contractual (Academia Nacional de Derecho y Ciencias Sociales de Córdoba). Disponible en http://www.acaderc.org.ar/doctrina/ articulos/artlorenzettiacademia

39 Mariana Bernal-Fandiño, El deber de coherencia en el derecho colombiano de los contratos, 54, 55 (Editorial Pontificia Universidad Javeriana, Bogotá, 2013). Disponible en: www.atlantiswordprocessor.com/en/forum/download.php?id=313 
De esta manera, la preparación del contrato se centra en establecer reglas que favorezcan la negociación de las prestaciones durante el tiempo en lugar de prever en el presente toda circunstancia que pudiera llegar a ser relevante en el futuro ${ }^{40}$.

\section{FUNCIÓN PROACTIVA DEL CONTRATO}

Las funciones analizadas en la primera parte de este texto convergen en la necesidad de entender y construir los contratos desde una nueva perspectiva.

Se ha planteado que la aproximación tradicional del derecho es una aproximación reactiva frente a los conflictos por lo que se proponen aproximaciones diferentes que le permitan al derecho y, en el caso que nos concierne, al derecho de los contratos tener una función distinta.

\section{A. E1 derecho preventivo como génesis de la función proactiva del contrato}

Se ha considerado que Louis Brown ${ }^{41}$, con sus trabajos en la década de los 50, es el "padre del derecho preventivo" y que la primera aproximación institucional alrededor de este tema se encuentra en la fundación del National Center of Preventive Law en $1986^{42}$.

Aplicando las reglas básicas de la medicina preventiva, el derecho contractual preventivo se enfoca en evitar el conflicto y buscar que el contrato sea realmente un instrumento de oportunidades y éxito para los contratantes.

Como lo señala Helena Haapio, las empresas no triunfan al ganar procesos $^{43}$. Los litigios son costosos, desgastantes, consumen tiempo y recursos que podrían utilizarse en producción y crecimiento.

40 Christian Courtis \& Nicolás Espejo, Por un "contrato de cohesión social": apuntes exploratorios, 12 (Comisión Económica para América Latina y el Caribe, CEPAL, Serie Políticas Sociales, División de Desarrollo Social, 129, Santiago de Chile, abril de 2007). Disponible en: http://biblioteca2012.hegoa.efaber.net/system/ebooks/17235/original/Por_un_contrato_de_cohesion_social.pdf

41 Louis Brown, Manual of Preventive Law (Prentice Hall, New York, 1950).

42 Edward A. Dauer, Four Principles for a Theory of Preventive Law, en A Proactive Approach to Contracting and Law, 13-33 (Helena HaAPIO, ed., Turku University Applied Sciences, TUAS, Turku, Finland, 2008).

43 Helena HaApio, Una onza de prevención...: asistencia jurídica proactiva para el éxito en la contratación corporativa, 1 Revista Foro Derecho Mercantil, 20, 7-45, 7 (2008). 
El prestigio de las compañías, sus mercados y su capacidad para prosperar y progresar pueden resultar destruidos antes de obtener algún beneficio producto de una decisión judicial.

El derecho contractual clásico es primordialmente un derecho remedial, no preventivo. Sin embargo, la idea de prevención no es ajena al derecho privado. En materia de derecho de daños, por ejemplo, ya se hace referencia a una función preventiva del daño, lo que resulta mucho más controversial teniendo en cuenta que tradicionalmente la función principal de la responsabilidad civil se ha centrado en su reparación. En el derecho societario, en particular con la aparición del fenómeno del gobierno corporativo ${ }^{44}$, se han desarrollado principios ${ }^{45}$ que buscan establecer buenas prácticas empresariales para prevenir la corrupción mediante una mayor transparencia y control de las compañías.

El derecho contractual no puede seguir concentrado en los efectos del litigio y en priorizar la necesidad de "blindar" a las partes frente a esa posibilidad; sino que debe buscar todas las herramientas necesarias para detectar problemas y prevenirlos en los acuerdos.

Edward A. Dauer ha identificado cuatro principios del derecho preventivo ${ }^{46}$ :

- Predicción del comportamiento humano. Se debe anticipar el comportamiento de las personas con el fin de evitar el litigio.

- Gestión de conflictos. Utilizando un paralelo con la medicina preventiva, se utiliza un tratamiento profiláctico de las situaciones.

- Postura frente a los riesgos. En lugar de enfocarse en la reducción de cada uno de los posibles riesgos, se deben analizar como un todo y reducirlos de manera conjunta.

- Servicio legal preventivo. Los abogados y consultores deben participar en la planeación del negocio desde el principio, para

44 Daniel Fischel, The Corporate Governance Movement, 35 Vanderbilt Law Review, 6, 1259-1292 (1982). Disponible en: http://chicagounbound.uchicago.edu/cgi/viewcontent.cgi?article=2419 \&context=journal_articles

45 Sobre estos principios, Organización para la Cooperación y el Desarrollo Económicos, OCDE, Principios de Gobierno Corporativo (OCDE, París, 2004). Disponible en: http://www.oecd.org/ corporate/ca/corporategovernanceprinciples/37191543.pdf

46 Edward A. Dauer, Four Principles for a Theory of Preventive Law, en A Proactive Approach to Contracting and Law, 13-33 (Helena HaApio, ed., Turku University Applied Sciences, TUAS, Turku, Finland, 2008). La traducción libre que se realiza en este texto obedece a los principios: Predicting human behavior, conflict management, embracement of risks, preventive legal service. 
prevenir los problemas que puedan presentarse en lugar de prestar los servicios cuando el conflicto ya existe.

El contrato es la base de los negocios y es el elemento central para llevarlos a cabo, pero los contratos son cada vez más difíciles y complejos. Estudios han evidenciado que los gerentes de las empresas ubican los asuntos jurídicos como uno de los retos más complejos de la administración moderna ${ }^{47}$.

Frente a esta realidad que no conoce fronteras, pues es una situación que como veremos es común en diferentes ordenamientos jurídicos, se ha propuesto una visión diferente. Esta nueva visión obedece a una función proactiva de los contratos, en la que tanto los contratos como los abogados deben ser un instrumento que añada valor a los negocios y no simplemente el último recurso al presentarse un conflicto.

Se desarrolla entonces una propuesta que va aún más allá de la función preventiva de los contratos: la función proactiva de los contratos.

\section{Gráfico 1}

\section{Función proactiva de los contratos}

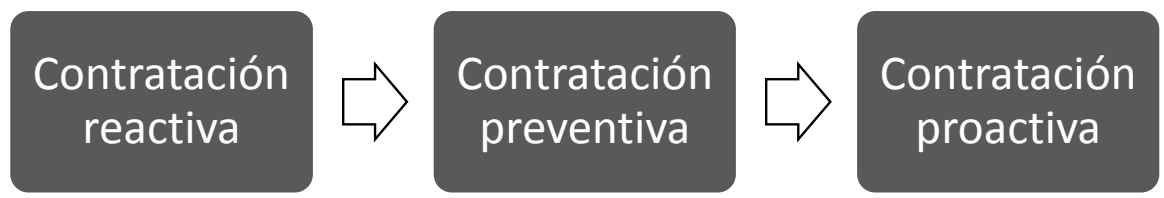

Fuente: elaboración propia

El derecho proactivo es de aparición reciente. Mientras en Europa se empezaba a discutir la función proactiva del derecho, en Estados Unidos el énfasis se ubicaba en el uso del derecho como una ventaja competitiva. Estos dos fenómenos, que se desarrolla-

47 Robert C. Bird, Law, Strategy, and Competitive Advantage, 44 Connecticut Law Review, 1, 63-97 (noviembre de 2011). Disponible en: http://uconn.lawreviewnetwork.com/files/2012/01/ Bird.pdf, http://works.bepress.com/robert_bird/2/ 
ron de forma paralela, han sido analizados en estudios de derecho comparado para encontrar las similitudes y sinergias entre ellos ${ }^{48}$.

El movimiento del derecho proactivo que tomó forma en los países nórdicos se ha extendido al resto de Europa. Su rápido crecimiento se debe, entre otras circunstancias, al apoyo del Comité Económico y Social Europeo (CESE) que rindió en 2008 dictamen sobre El planteamiento proactivo del Derecho: un paso más hacia una mejor legislación ${ }^{49}$. Según este dictamen:

Desde hace demasiado tiempo, el acento, en el ámbito jurídico, ha recaído en el pasado. Los legisladores y la judicatura han reaccionado a lagunas, litigios, plazos no respetados e infracciones, intentando resolverlos y remediarlos. Los litigios, los procedimientos y los recursos para imponer el respeto de las normas cuestan demasiado, y ese coste no puede medirse solamente en términos monetarios.

El CESE propugna un cambio de paradigma. Ha llegado el momento de abandonar el secular enfoque reactivo del Derecho y adoptar un planteamiento proactivo. Es hora de considerar el Derecho de una forma diferente: mirar hacia adelante y no hacia atrás, centrarse en cómo se utiliza y cómo actúa el Derecho en la vida diaria y cómo es recibido en la comunidad que pretende regular. Si bien responder a los problemas y resolverlos sigue siendo importante, prevenir las causas de los problemas es vital, como lo es también satisfacer las necesidades de los ciudadanos y empresas y facilitar su interacción productiva.

En Estados Unidos, con una aproximación similar, se ha explorado la función del derecho como una herramienta de competitividad $^{50}$.

Si bien el derecho proactivo se basa en el derecho preventivo, le añade una nueva dimensión constructiva que promueve un mejor comportamiento entre los sujetos. Desde una aproximación proactiva, el derecho de contratos debe procurar la colaboración de los asesores legales en todo el proceso del negocio por medio de grupos

48 George J. Siedel \& Helena Haapio, Using Proactive Law for Competitive Advantage (Ross School of Business, Working Paper No. 1148, University of Michigan, agosto de 2010). Disponible en: http://deepblue.lib.umich.edu/bitstream/handle/2027.42/77601/1148_siedel. pdf?sequence $=1$

49 Comité Económico y Social Europeo, CESE, Dictamen CESE 1905/2008, El planteamiento proactivo del Derecho: un paso más hacia una mejor legislación. Disponible en: https://dm.eesc. europa.eu/EESCDocumentSearch/Pages/opinionssearch.aspx

50 George Siedel \& Helena HaApio, Proactive Law for Managers. A Hidden Source of Competitive Advantage (Gower Publishing, Farnham, Surrey, United Kingdom, 2011). 
interdisciplinares. El abogado debe trabajar conjuntamente con los otros profesionales involucrados (gerentes, ingenieros, economistas, etc.) para optimizar los resultados del acuerdo. Las personas a quienes atañe el contrato deben estar motivadas y comprometidas con el acuerdo y para lograrlo, lo principal es entenderlo, tener claras las reglas y crear mecanismos para que su cumplimiento sea fácil. Para ello, la implementación de medidas que otorguen flexibilidad en los contratos puede ser de gran utilidad.

Helena Haapio ha explicado que el derecho proactivo debe procurar el autocuidado del cliente, al trabajar en tres niveles principales para poder aprovechar todas las oportunidades y ventajas que tienen los contratos:

\section{Gráfico 2}

\section{Tres niveles principales de asistencia jurídica proac- tiva en la contratación corporativa}

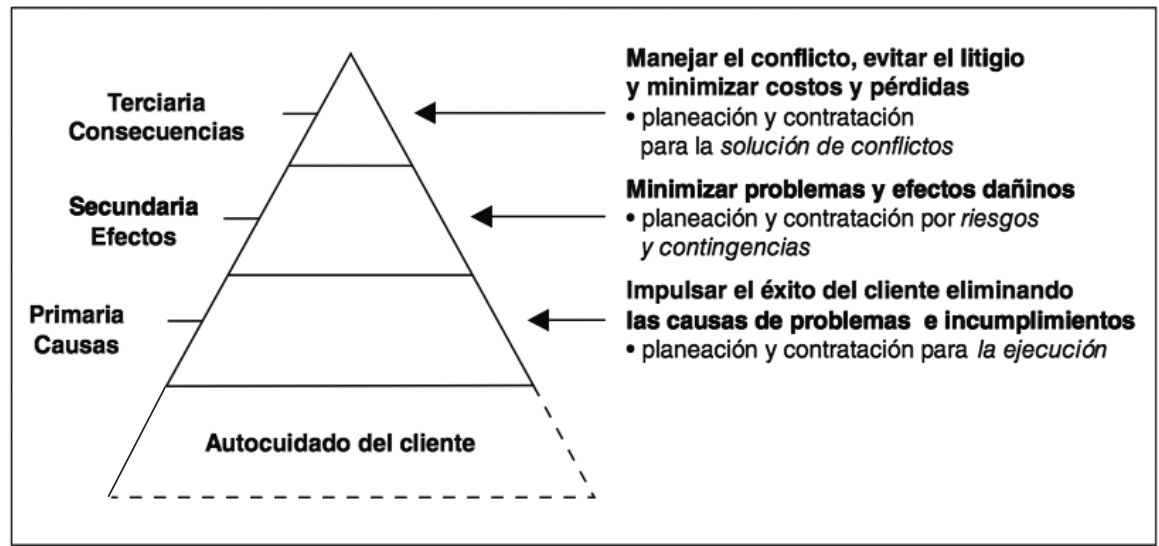

Fuente: tomado de Helena HaApIo, Una onza de prevención...: asistencia jurídica proactiva para el éxito en la contratación corporativa, 1 Revista Foro Derecho Mercantil, 20, 7-45, 10 (2008).

El gráfico 2 ilustra la forma en que debe prepararse un contrato de modo que en primer lugar, en la base de la pirámide, está la fase de impulso del negocio en la que se deben plasmar en el contrato los objetivos que aseguren el éxito del negocio y los resultados deseados. En un segundo nivel, se deben redactar las cláusulas relacionadas con la distribución de riesgos, las indemnizaciones por incumpli- 
miento, entre otras. $\mathrm{Y}$ en un tercer nivel se deben establecer los mecanismos de resolución de conflictos.

En la actualidad, los contratos presentan problemas de fondo $\mathrm{y}$ de forma. Los problemas de fondo se manifiestan, como se ha mencionado, por el énfasis que se le otorga a todo lo que puede salir mal en el negocio en lugar de ser una herramienta que se concentre en que las partes obtengan los mejores beneficios. Por lo general, esta situación se presenta pues la aproximación del contrato es exclusivamente legal en lugar de tener en cuenta otras perspectivas como la económica y la humana ${ }^{51}$.

Acerca de los problemas de forma de los contratos, puede decirse que en su mayoría consisten en la complejidad de la redacción y visualización de los contratos contemporáneos.

En efecto, los contratos deberían ser entendidos por sus operadores. No pueden convertirse en un terreno exclusivo de los abogados. Los contratos hoy no se entienden, son extensos y se utiliza un lenguaje complejo que confunde incluso a los mismos abogados.

Se genera entonces un abismo entre quienes redactan los contratos y quienes deben ejecutarlos. En una empresa, el gerente, los administradores, los economistas, los ingenieros deben conocer y entender los compromisos que adquieren y su alcance. Pero la forma en que están redactados los acuerdos imposibilita esta simple necesidad. Los contratos son para los abogados, un mal necesario (¿los contratos?, ¿los abogados? tal vez ambos) al que debe acudirse en caso de problemas.

El Comité Económico y Social Europeo, CESE — que, como se ha mencionado, ha adoptado la visión proactiva del derecho-, explica que el derecho desde este punto de vista debe capacitar y facultar tanto a las personas como a las empresas para que conozcan sus derechos y responsabilidades y puedan alcanzar sus objetivos ${ }^{52}$.

En cuanto al derecho del consumo, los usuarios raramente leen los contratos que firman y de hacerlo, realmente no entienden su contenido. Frente a esta situación, el US Consumer Financial

51 Thomas D. Barton, Improving Contracts through Expanding Perspectives of Understanding, en Proceedings of the 2013 Academic Forum on Integrating Law and Contract Management: Proactive, Preventive and Strategic Approaches (2013).

52 Comité Económico y Social Europeo, CESE, Dictamen CESE 1905/2008, El planteamiento proactivo del Derecho: un paso más hacia una mejor legislación. Disponible en: https://dm.eesc. europa.eu/EESCDocumentSearch/Pages/opinionssearch.aspx 
Protection Bureau (CFPB), el ente de protección al consumidor en materia financiera en Estados Unidos, lanzó un proyecto sobre la posibilidad de simplificar los contratos de tarjetas de crédito ${ }^{53}$. Esta iniciativa surgió a raíz de un estudio de J. D. Power ${ }^{54}$, según el cual los usuarios de tarjetas de crédito no entienden estos acuerdos, no solamente por el tipo de letra que se utiliza, sino por la forma en que están redactados ${ }^{55}$.

En la contratación proactiva, se deben considerar dos dimensiones. Una dimensión interpartes, en la que la colaboración y la focalización en la realización de los objetivos de los contratantes constituyen el eje principal de la preparación del acuerdo, y otra dimensión que se aplica a los contratos con personas jurídicas y es la aproximación interdisciplinar en la construcción de los contratos. La primera dimensión se acerca a la perspectiva de la función social del contrato y al deber de cooperación de los contratantes. La segunda dimensión involucra la colaboración pero en la propia empresa y la necesidad de construir los contratos más allá de la estricta visión jurídica, teniendo en cuenta aspectos económicos, personales, ambientales, etc., de manera que los contratos sean aplicados y entendidos por quienes deben utilizarlos.

\section{B. La visualización de los contratos}

Los contratos, como cualquier otro documento legal, deberían redactarse teniendo en cuenta su uso y facilidad para los usuarios de la misma forma como se diseña un producto, un artefacto que sea sencillo de utilizar y entender.

En su mayoría, los contratos utilizan un lenguaje demasiado técnico, son excesivamente largos y se enfocan en la responsabilidad derivada del incumplimiento de las obligaciones contractuales en

53 Con este prototipo de contrato, se separa de forma clara para el usuario la información más relevante en materia de precios, riesgos, plazos y se maneja un lenguaje más sencillo en general. US Consumer Financial Protection Bureau, CFPB, CFPB Aims to Simplify Credit Card Agreements (December 7, 2011). Disponible en http://www.consumerfinance.gov/newsroom/ consumer-financial-protection-bureau-aims-to-simplify-credit-card-agreements/

54 J. D. Power Studies, 2014 U.S. Credit Card Satisfaction Study. Disponible en: http://www. jdpower.com/resource/us-credit-card-satisfaction-study

55 Sobre los problemas detectados en los contratos de tarjetas de crédito en Estados Unidos, Connie Prater, U.S. Credit Card Agreements Unreadable to 4 out of 5 Adults. Contracts Written at a Reading Level Most Can't Understand. Disponible en: CreditCards.com: http:// www.creditcards.com/credit-card-news/credit-card-agreement-readability-1282.php 
lugar de ser una guía para su cumplimiento ${ }^{56}$. Por estas razones, entre otras, el mal entendimiento de los contratos y su equivocada aproximación ocasionan problemas en su ejecución.

Si los contratantes no leen los contratos o no los entienden, los contratos pierden su función. El contrato no puede ser una mera formalidad o un documento al cual acudir en caso de dificultades. El contrato debe ser una carta de navegación y una oportunidad de planeación e innovación para la obtención de resultados óptimos en los negocios.

El movimiento hacia la visualización de los contratos pretende hacerlos más simples y amables. Añadir gráficas y cuadros puede transformar pesados documentos legales tradicionales en instrumentos de colaboración, innovación, planeación estratégica y valor social ${ }^{57}$.

\section{Caso simplificación de contratos celulares por parte de la CRC}

La Comisión de Regulación de Comunicaciones de Colombia, CRC, lanzó al comenzar el año 2014 un novedoso proyecto sobre simplificación de los contratos de telefonía móvil celular. Después de varios meses de socialización de esta iniciativa, la CRC expidió la resolución 4625 de $2014^{58}$ que establece unos modelos de contrato único para la prestación de servicios por redes móviles.

56 Thomas D. Barton, Gerlinde Berger-Walliser \& Helena HaApio, Visualization: Seeing Contracts for What They Are, and What They Could Become, 19 Journal of Law, Business \& Ethics, 47-64 (2013). Disponible en: http://www.yasni.info/ext.php?url=http $\% 3 \mathrm{~A} \% 2 \mathrm{~F} \% 2 \mathrm{Fsch}$ olarlycommons.law.cwsl.edu $\% 2 \mathrm{Fcgi} \% 2 \mathrm{Fviewcontent.cgi} \% 3$ Farticle $\% 3 \mathrm{D} 1008 \% 26 \mathrm{amp} \% 3 \mathrm{Bc}$ ontext $\% 3$ Dfs \&name $=$ Gerlinde + Berger\&showads $=1 \& l c=e s-e s \& l g=e s \& r g=u s \& r i p=c o, h t t p: / /$ papers.ssrn.com/sol3/papers.cfm?abstract_id=2005434

57 Thomas D. Barton, Gerlinde Berger-Walliser \& Helena Haapio, Visualization: Seeing Contracts for What They Are, and What They Could Become, 19 Journal of Law, Business \& Ethics, 47-64 (2013). Disponible en: http://www.yasni.info/ext.php?url=http $\% 3 \mathrm{~A} \% 2 \mathrm{~F} \% 2 \mathrm{Fsch}$ olarlycommons.law.cwsl.edu $\% 2 \mathrm{Fcgi} \% 2$ Fviewcontent.cgi $\% 3$ Farticle $\% 3 \mathrm{D} 1008 \% 26 \mathrm{amp} \% 3 \mathrm{Bc}$ ontext $\% 3$ Dfs \&name $=$ Gerlinde + Berger \&showads $=1 \& l c=e s-e s \& l g=e s \& r g=u s \& r i p=c o, h t t p: / /$ papers.ssrn.com/sol3/papers.cfm?abstract_id=2005434

58 Comisión de Regulación de Comunicaciones de Colombia, CRC, Resolución 4625 de 2014, por la cual se establecen los modelos de contrato único y de las condiciones generales, de prestación de servicios provistos a través de redes móviles, se modifica la Resolución CRC 3066 de 2011 y se dictan otras disposiciones, 31 de octubre de 2014. Disponible en: https:// www.crcom.gov.co/es/noticia/resoluci-n-crc-4625-de-2014 
Esta apuesta novedosa de la CRC surgió como consecuencia de un estudio que evidenció que los usuarios de telefonía móvil celular no leen los contratos que firman y, si lo hacen, no los entienden ${ }^{59}$. El estudio mencionado realizó un análisis de la comunicación visual de los contratos de telefonía móvil actuales en Colombia. Esta revisión desde el punto visual y gráfico de los contratos pretende determinar si los acuerdos que se están celebrando con los usuarios constituyen una fuente de comunicación que efectivamente les permita conocer claramente el servicio que están contratando y sus condiciones.

En el análisis se tuvieron en cuenta diferentes indicadores como los títulos, la navegabilidad del documento, los espacios en blanco, la consistencia, la densidad del contenido, el énfasis en elementos de importancia, la organización del contenido, la limpieza en la implementación gráfica, los separadores, las márgenes. La escala de evaluación que se utilizó es la siguiente:

Insuficiente: no cubre ninguno de los criterios sugeridos.

Pobre: contiene algunos de los elementos pero su aplicación es deficiente y/o errónea.

Satisfactorio: se presentan los criterios mínimos de aplicación de los elementos.

Bueno: se contienen varios de los elementos sugeridos con buen criterio y aplicado de manera adecuada.

Muy bueno: el documento presenta atención y resolución a todos los elementos sugeridos.

El resultado obtenido se expresó en el documento soporte con el gráfico 3:

59 Ver este estudio en el documento soporte de la resolución, Comisión de Regulación de Comunicaciones de Colombia, CRC, Simplificación de modelos de contrato de telefonía móvil. Documento soporte. Disponible en: http://www.crcom.gov.co/recursos_user/Documentos_CRC_2014/ Actividades_Regulatorias/Simplificacion/Documento_soporte_simplificacion.pdf 


\section{Gráfico 3}

\section{Análisis de la comunicación visual}

\section{ANÁLISIS DE COMUNICACIÓN VISUAL}

GRÁFICA COMPARATIVA

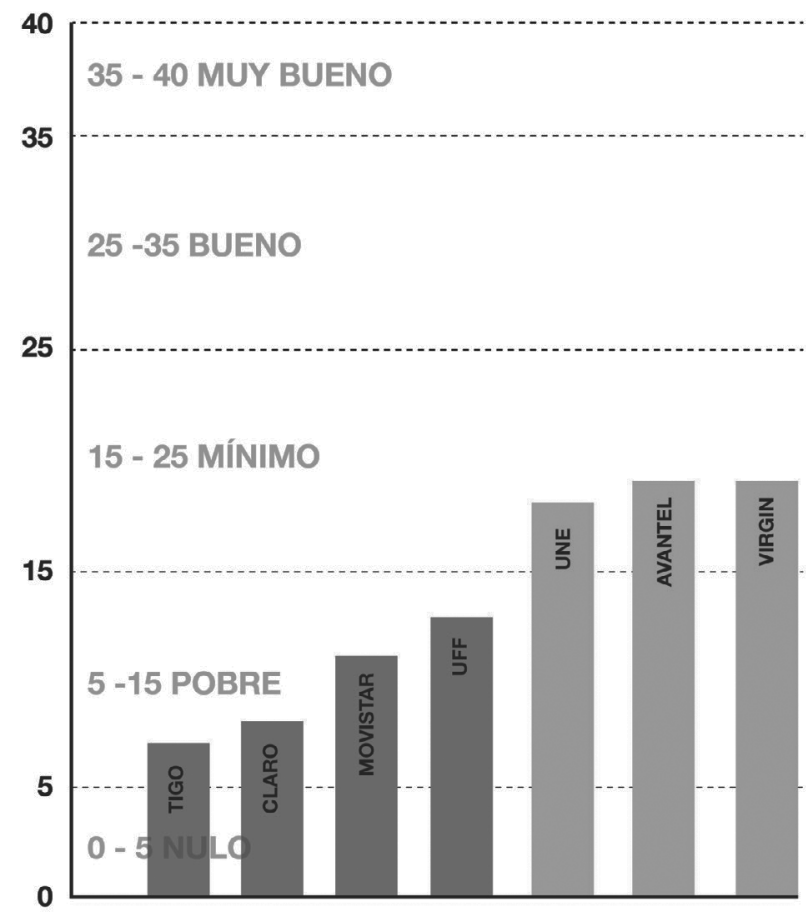

Fuente: Comisión de Regulación de Comunicaciones

Del gráfico 3 se puede apreciar que la mayoría de los operadores de telefonía móvil tiene una pobre visualización en sus contratos y solo algunos de ellos cumplen los estándares mínimos.

Con base en esta observación, la CRC evidenció la necesidad de una transformación para favorecer las relaciones entre proveedores y usuarios. Se requería un contrato que permitiera a los consumidores entender y ejercer sus derechos.

Por medio de su resolución 4625 de 2014, la CRC regula el contenido y el formato de estos contratos de manera que los proveedores de redes y servicios de telecomunicaciones móviles deben adoptar 
estrictamente los modelos establecidos por el regulador, ya que estos han sido diseñados para ser claros y sencillos para los usuarios.

De lo anterior queremos resaltar dos aspectos. El primero consiste en la posibilidad del regulador de imponer un contrato tipo para las partes, lo que implica un límite a la autonomía privada. Esta posibilidad puede relacionarse con la forma como la mayor parte de la doctrina brasileña ha entendido el alcance de la función social del contrato establecida en la legislación de ese país. El pilar de la autonomía privada se tiene que adaptar a una forma más real de contratación, de manera que el derecho no insista en la observancia de unas reglas que parten de una base de igualdad y libertad inexistentes, sino que parte de la realidad de los usuarios y busca la mejor solución para que el contrato cumpla verdaderamente su finalidad.

En segundo lugar, es sorprendente y loable, en nuestra opinión, que sea precisamente una entidad pública la pionera en nuestro país en proponer una visión proactiva de los contratos, pues si bien en Colombia se han realizado importantes avances en materia de protección al consumidor, la imposición a las empresas de un contrato simplificado y visualmente claro es algo realmente novedoso en cualquier ordenamiento jurídico. 


\section{CONCLUSIONES}

Muchas de las inquietudes alrededor de la contratación contemporánea, en particular en lo referente a la función de los contratos en la sociedad, encuentran una respuesta en la contratación proactiva.

La contratación proactiva busca generar diálogos entre los distintos agentes que intervienen en la contratación y sus distintos intereses. Se debe ampliar la visión del contrato para generar mejores prácticas en la planeación, el diseño, la comunicación y la implementación de los acuerdos.

Un ejemplo de contratación proactiva se encuentra en la reciente resolución 4625 de 2014 de la CRC. Por medio de esta regulación, vemos cómo convergen elementos de la contratación proactiva y de la función social y económica de los contratos. En efecto, se propende por unos contratos eficientes, que sean un instrumento real y no únicamente formal para las partes. Adicionalmente, la propuesta en sí es reflejo de una visión contractual que va más allá de un acuerdo de dos voluntades pues configura una relación social que se encuentra dentro de un mercado regulado y que, por lo tanto, está construido con elementos tanto consensuales como no consensuales.

Desde un punto de vista estrictamente jurídico, la aproximación tradicional del derecho de los contratos debe complementarse con otras visiones para que el contrato se convierta en una herramienta eficiente que responda a las verdaderas necesidades de los contratantes y del tráfico jurídico y pueda cumplir así su finalidad en la sociedad. 


\section{BIBLIOGRAFÍA}

\section{Libros}

Bernal-Fandiño, Mariana, El deber de coherencia en el derecho colombiano de los contratos (Editorial Pontificia Universidad Javeriana, Bogotá, 2013). Disponible en: www.atlantiswordprocessor.com/en/forum/download.php?id=313

Betti, Emilio, Teoría general de las obligaciones (José Luis de los Mozos, trad., Editorial Revista de Derecho Privado, Madrid, 1969).

Bianca, Massimo, Derecho civil, el contrato (Fernando Hinestrosa \& Édgar Cortés, trads., Editorial Universidad Externado de Colombia, Bogotá, 2007).

Brown, Louis, Manual of Preventive Law (Prentice Hall, New York, 1950).

Cooter, Robert \& Ulen, Thomas, Law and Economics (Pearson/Addison-Wesley, Boston, 2004).

Courtis, Christian \& Espejo, Nicolás, Por un "contrato de cohesión social": apuntes exploratorios (Comisión Económica para América Latina y el Caribe, CEPAL, Serie Políticas Sociales, División de Desarrollo Social, 129, Santiago de Chile, abril de 2007). Disponible en: http://biblioteca2012.hegoa.efaber.net/system/ ebooks/17235/original/Por_un_contrato_de_cohesion_social.pdf

MacNeil, Ian Roderick, The New Social Contract. An Inquiry into Modern Contractual Relations (Yale University Press, New Haven, Connecticut, 1980).

Messineo, Francesco, Doctrina general del contrato, Tomo I (Rodolfo Fontanarrosa, Santiago Sentis-Melendo \& M. Volterra, trads., Ediciones Jurídicas EuropaAmérica, Buenos Aires, 1952).

Ospina-Fernández, Guillermo, Teoría general del contrato y de los demás actos jurídicos o negocios jurídicos (4a ed., Editorial Temis, Bogotá, 1994).

SChäFER, HANs-BERnd \& OTt, Claus, Manual de análisis económico del derecho (Editorial Tecnos, Madrid, 1991).

Siedel, George \& Hadpio, Helena, Proactive Law for Managers. A Hidden Source of Competitive Advantage (Gower Publishing, Farnham, Surrey, United Kingdom, 2011).

\section{Contribuciones en obras colectivas}

Barton, Thomas D., Improving Contracts through Expanding Perspectives of Understanding, en Proceedings of the 2013 Academic Forum on Integrating Law and Contract Management: Proactive, Preventive and Strategic Approaches (2013).

Boutonnet, Mathilde, L'efficacité environnementale du contrat, en L'efficacité du droit de l'environnement - Mise en cuvre et sanctions, 21-46 (Olivera BošKovi囚, dir., Éditions Dalloz, Paris, 2010).

Dauer, Edward A., Four Principles for a Theory of Preventive Law, en A Proactive Approach to Contracting and Law, 13-33 (Helena HaAPIo, ed., Turku University Applied Sciences, TUAS, Turku, Finland, 2008). 
Moreno-VÁsquez, Héctor Andrés, Contratación sostenible, en Perspectivas del derecho ambiental en Colombia, 390-408 (Beatriz Londoño-Toro, Gloria Amparo Rodríguez, Giovanni J. Herrera-Carrascal, eds., Editorial Universidad del Rosario, Bogotá, 2006).

\section{Revistas}

Barroso, Lucas Abreu, Función ambiental de los contratos agrarios, 1 Rivista di Diritto Agrario, Milano, 190-202 (2005). Disponible en: http://www.abda.com.br/ palestrabarroso.htm, http://www.academia.edu/5427909/Funci\%C3\%B3n_ ambiental_de_los_contratos_agrarios

Barton, Thomas D.; Berger-Walliser, Gerlinde \& HaApio, Helena, Visualization: Seeing Contracts for What They Are, and What They Could Become, 19 Journal of Law, Business \& Ethics, 47-64 (2013). Disponible en: http://www.yasni.info/ext.php ?url=http $\% 3 \mathrm{~A} \% 2 \mathrm{~F} \% 2 \mathrm{Fscholarlycommons.law.cwsl.edu} \% 2 \mathrm{Fcgi} \% 2 \mathrm{Fviewcontent.}$ cgi $\% 3$ Farticle $\% 3 \mathrm{D} 1008 \% 26 \mathrm{amp} \% 3 \mathrm{Bcontext} \% 3 \mathrm{Dfs} \&$ name $=$ Gerlinde+Berger\&s howads $=1 \& l \mathrm{c}=\mathrm{es}-\mathrm{es} \& \lg =\mathrm{es} \& \mathrm{rg}=$ us\&rip=co, http://papers.ssrn.com/sol3/papers. cfm?abstract_id=2005434

Bird, Robert C., Law, Strategy, and Competitive Advantage, 44 Connecticut Law Review, 1, 63-97 (noviembre de 2011). Disponible en: http://uconn.lawreviewnetwork.com/ files/2012/01/Bird.pdf, http://works.bepress.com/robert_bird/2/

Branco, Gerson Luiz Carlos, Libertad contractual y su funcionalización: orientación metodológica y lenguaje utilizados por la comisión elaboradora del Código Civil brasileño, 123 Vniversitas, 347-372 (julio-diciembre de 2011). Disponible en: http:// www.scielo.org.co/pdf/vniv/n123/n123a12.pdf

Coase, Ronald H., The Problem of Social Cost, 3 Journal of Law and Economics, 1-44 (1960). Disponible en: http://www2.econ.iastate.edu/classes/tsc220/hallam/ Coase.pdf

DiMatteo, Larry A., Strategic Contracting: Contract Law as a Source of Competitive Advantage, 47 American Business Law Journal, 4, $727-794$ (2010). Disponible en: http://works.bepress.com/larry_dimatteo/3/

Eastman, Wayne, How Coasean Bargaining Entails a Prisoners' Dilemma, 72 Notre Dame Law Review, 1, 89-101 (1996). Disponible en: http://scholarship.law.nd.edu/ ndlr/vol72/iss1/3/

Eisenberg, Melvin Aron, The Emergence of Dynamic Contract Law, 88 California Law Review, 6, 1743-1814 (December, 2000). Disponible en: http://scholarship.law. berkeley.edu/cgi/viewcontent.cgi?article $=1470 \&$ context $=$ californialawreview

Fischel, Daniel, The Corporate Governance Movement, 35 Vanderbilt Law Review, 6, 1259-1292 (1982). Disponible en: http://chicagounbound.uchicago.edu/cgi/ viewcontent.cgi?article $=2419 \&$ context $=$ journal_articles

HaApio, Helena, Una onza de prevención...: asistencia jurídica proactiva para el éxito en la contratación corporativa, 1 Revista Foro Derecho Mercantil, 20, 7-45 (2008).

Hayer, Friedrich August von, Economics and Knowledge, 4 Economica, New Series, 13, 33-54 (February, 1937). Disponible en: http://www.econlib.org/library/ NPDBooks/Thirlby/bcthLS3.html 
Kronman, Anthony T., Mistake, Disclosure, Information, and the Law of Contracts, 7 Journal of Legal Studies, 1, 1-34 (1978). Disponible en: http://www.rwi.uzh.ch/ lehreforschung/alphabetisch/weberr/archiv/FS08/unterlagen/unterlagenLE/ aufsaetze/MistakeDisclosureInformationandtheLawofContracts.pdf

MacNeil, Ian Roderick, The Many Futures of Contracts, 47 Southern California Law Review, 691-816 (1974).

Markovits, Daniel, Contract and Collaboration, 113 Yale Law Journal, 1417-1518 (2004). Disponible en: http://digitalcommons.law.yale.edu/fss_papers/414/, http:// digitalcommons.law.yale.edu/cgi/viewcontent.cgi?article $=1413 \&$ context $=$ fss_ papers

Poli, Luciana Costa \& Hazan, Bruno Ferraz, A função social dos contratos como instrumento para a sustentabilidade, 15 Revista Direito e Liberdade, RDL, ESMARN, 1, 171-193 (2013). Disponible en: http://www.esmarn.tjrn.jus.br/ revistas/index.php/revista_direito_e_liberdade/article/viewFile/550/512

Plata-López, Luis Carlos, La naturaleza social y económica del contrato, 23 Revista de Derecho, Universidad del Norte, 97-111 (2005). Disponible en: http://www.redalyc. org/articulo.oa? $\mathrm{id}=85102305$

Salvador-Coderch, Pablo, Alteración de circunstancias en el art. 1213 de la propuesta de modernización del Código Civil en materia de obligaciones y contratos, 4 InDret, Revista para el Análisis del Derecho, 1-60 (octubre de 2009). Disponible en: http:// www.indret.com/pdf/687_es.pdf

Solarte-Rodríguez, Arturo, La buena fe contractual y los deberes secundarios de conducta, 108 Vniversitas, 281-315 (diciembre de 2004). Disponible en: http://www. javeriana.edu.co/revistas/Facultad/juridicas/universitas/UserFiles/Descargas/ ediciones/108/7_Solarte.pdf

Stigler, George, The Economics of Information, 69 The Journal of Political Economy, 3, 213-225 (June, 1969). Disponible en: http://home.uchicago.edu/ vlima/courses/ econ200/spring01/stigler.pdf

Timm, Luciano Benetti, La función social del derecho contractual en el Código Civil brasileño: justicia distributiva vs. eficiencia económica, 52 Revista de Instituciones, Ideas y Mercados, 5-51 (2010). Disponible en: http://www.eseade.edu.ar/files/riim/ RIIM_52/52_1_benetti_timm.pdf

Wilches-Durán, Rafael E., Principio ambiental de precaución y contratación mercantil en derecho colombiano. Planteamiento del problema, 123 Vniversitas, 283-313 (2011). Disponible en: http://www.redalyc.org/articulo.oa?id=82522608010, http:// vniversitasjuridica.javeriana.edu.co/descargas.php?archivo=Universitas $-123 \% 20$ $-\% 209$. pdf\&idArt $=313 \&$ edicion $=123$ 


\section{Working papers}

Siedel, George J. \& Haapio, Helena, Using Proactive Law for Competitive Advantage (Ross School of Business, Working Paper No. 1148, University of Michigan, agosto de 2010). Disponible en: http://deepblue.lib.umich.edu/bitstream/ handle/2027.42/77601/1148_siedel.pdf?sequence=1

\section{Documentos, informes, publicaciones online, reportes}

Colombia, Ministerio de Ambiente, Vivienda y Desarrollo Territorial, Política Nacional de Producción y Consumo Sostenible (Ministerio de Ambiente, Vivienda y Desarrollo Territorial, Bogotá, 2010). Disponible en: http://www.soyecolombiano.com/site/ Portals/0/documents/PoliticaNacionaldeProduccionyConsumoSostenible.pdf

Comité Económico y Social Europeo, CESE, Dictamen CESE 1905/2008, El planteamiento proactivo del Derecho: un paso más hacia una mejor legislación. Disponible en: https://dm.eesc.europa.eu/EESCDocumentSearch/Pages/ opinionssearch.aspx

J. D. Power Studies, 2014 U.S. Credit Card Satisfaction Study. Disponible en: http:// www.jdpower.com/resource/us-credit-card-satisfaction-study

Lorenzetti, Ricardo Luis, Problemas actuales de la teoría contractual (Academia Nacional de Derecho y Ciencias Sociales de Córdoba). Disponible en http:// www.acaderc.org.ar/doctrina/articulos/artlorenzettiacademia

Prater, Connie, U.S. Credit Card Agreements Unreadable to 4 out of 5 Adults. Contracts Written at a Reading Level Most Can't Understand. Disponible en CreditCards. com: http://www.creditcards.com/credit-card-news/credit-card-agreementreadability-1282.php

Secretaría Distrital de Ambiente, Programa de Gestión Ambiental Empresarial. Disponible en: http://ambientebogota.gov.co/es/web/gae/inicio

US Consumer Financial Protection Bureau, CFPB, CFPB Aims to Simplify Credit Card Agreements (December 7, 2011). Disponible en http://www.consumerfinance. gov/newsroom/consumer-financial-protection-bureau-aims-to-simplify-creditcard-agreements/

\section{Normatividad colombiana}

Código Civil, Ley 57 de 1887. Disponible en: http://www.secretariasenado.gov.co/senado/ basedoc/codigo_civil.html

Código de Comercio, Decreto 410 de 1971. Disponible en: http://www.alcaldiabogota. gov.co/sisjur/normas/Norma1.jsp?i=41102

Comisión de Regulación de Comunicaciones de Colombia, CRC, Resolución 4625 de 2014, por la cual se establecen los modelos de contrato único y de las condiciones generales, de prestación de servicios provistos a través de redes móviles, se 
modifica la Resolución CRC 3066 de 2011 y se dictan otras disposiciones, 31 de octubre de 2014. Disponible en: https://www.crcom.gov.co/es/noticia/resolucin-crc-4625-de-2014

Comisión de Regulación de Comunicaciones de Colombia, CRC, Simplificación de modelos de contrato de telefonía móvil. Documento soporte. Disponible en: http://www.crcom.gov.co/recursos_user/Documentos_CRC_2014/Actividades_ Regulatorias/Simplificacion/Documento_soporte_simplificacion.pdf

Concejo de Bogotá, Acuerdo 540 de 2013, por medio del cual se establecen los lineamientos del programa distrital de compras verdes y se dictan otras disposiciones, 26 de diciembre de 2013. Disponible en: http://www.alcaldiabogota. gov.co/sisjur/normas/Norma1.jsp?i=56074

\section{Normatividad internacional}

Brasil, Código Civil, Ley 10406 del 10 de enero de 2002. Disponible en: http://www. wipo.int/wipolex/es/details.jsp?id=9615

Organización para la Cooperación y el Desarrollo Económicos, OCDE, Principios de Gobierno Corporativo (OCDE, París, 2004). Disponible en: http://www.oecd.org/ corporate/ca/corporategovernanceprinciples/37191543.pdf

\section{Tratados internacionales}

Conferencia de las Naciones Unidas sobre el Medio Ambiente y el Desarrollo, Declaración de Río de Janeiro en 1992 sobre el Medio Ambiente y el Desarrollo, 3 al 14 de junio de 1992. Disponible en: http://www.un.org/spanish/esa/sustdev/ agenda21/riodeclaration.htm

Plan de Aplicación de las Decisiones de la Cumbre Mundial sobre el Desarrollo Sostenible, Johannesburgo (Sudáfrica), 2 al 4 de septiembre de 2002. Disponible en: http://www.revistafuturos.info/documentos/johanesburgo/a_conf199_L1.doc. 
